\title{
Introduction to Higher-Order Iterative Methods for Finding Multiple Roots of Nonlinear Equations
}

\author{
Rajinder Thukral \\ Padé Research Centre, 39 Deanswood Hill, Leeds, West Yorkshire LS17 5JS, UK \\ Correspondence should be addressed to Rajinder Thukral; rthukral@hotmail.co.uk
}

Received 28 September 2012; Accepted 19 November 2012

Academic Editor: Petr Ekel

Copyright (C) 2013 Rajinder Thukral. This is an open access article distributed under the Creative Commons Attribution License, which permits unrestricted use, distribution, and reproduction in any medium, provided the original work is properly cited.

We introduce two higher-order iterative methods for finding multiple zeros of nonlinear equations. Per iteration the new methods require three evaluations of function and one of its first derivatives. It is proved that the two methods have a convergence of order five or six.

\section{Introduction}

Solving nonlinear equations is one of the most important problems in numerical analysis. In this paper, we consider iterative methods to find a multiple root $\alpha$ of multiplicity $m$, that is, $f^{(j)}(\alpha)=0, j=0,1, \ldots, m-1$, and $f^{(m)}(\alpha) \neq 0$, of a nonlinear equation

$$
f(x)=0 \text {, }
$$

where $f: I \subset \mathbb{R} \rightarrow \mathbb{R}$ is a scalar function on an open interval $I$ and it is sufficiently smooth in a neighbourhood of $\alpha$. In recent years, some modifications of the Newton method for multiple roots have been proposed and analysed [1-8]. However, there are not many methods known to handle the case of multiple roots. Hence we present two higher-order methods for finding multiple zeros of a nonlinear equation and only use four evaluations of the function per iteration. In addition, the new methods have a better efficiency index than the third- and fourth-order methods given in $[1-3,7]$; in view of this fact, the new methods are significantly better when compared with the established methods.

The well-known Newton's method for finding multiple roots is given by

$$
x_{n+1}=x_{n}-m \frac{f\left(x_{n}\right)}{f^{\prime}\left(x_{n}\right)},
$$

which converges quadratically [4]. For the purpose of this paper, we use (2) to construct new higher-order methods.

\section{Development of the Methods and Convergence Analysis}

In this section, we define new higher-order methods. In order to establish the order of convergence of the new methods we state the three essential definitions.

Definition 1. Let $f(x)$ be a real function with a simple root $\alpha$, and let $\left\{x_{n}\right\}$ be a sequence of real numbers that converge towards $\alpha$. The order of convergence $p$ is given by

$$
\lim _{n \rightarrow \infty} \frac{x_{n+1}-\alpha}{\left(x_{n}-\alpha\right)^{p}}=\zeta \neq 0,
$$

where $\zeta$ is the asymptotic error constant and $p \in \mathbb{R}^{+}$.

Definition 2. Let $k$ be the number of function evaluations of the new method. The efficiency of the new method is measured by the concept of efficiency index $[9,10]$ and defined as

$$
p^{1 / k}
$$

where $p$ is the order of convergence of the method.

Definition 3. Kung-Traub conjectured that the multipoint iterative methods without memory, requiring $k+1$ function evaluations per iteration, have the order of convergence of at most

$$
p=2^{k}
$$


Multipoint methods that satisfy the Kung-Traub conjecture are usually called optimal methods [11].

Recently, Thukral [8] presented a fourth-order iterative method, given by

$$
\begin{gathered}
y_{n}=x_{n}-m \frac{f\left(x_{n}\right)}{f^{\prime}\left(x_{n}\right)}, \\
x_{n+1}=x_{n}-m\left(\sum_{i=1}^{3} i\left(\frac{f\left(y_{n}\right)}{f\left(x_{n}\right)}\right)^{i / m}\right)\left(\frac{f\left(x_{n}\right)}{f^{\prime}\left(x_{n}\right)}\right) .
\end{gathered}
$$

In fact, the new methods presented in this paper are the extension of the above scheme. To develop the higher-order methods, we use the above two steps and introduce the third step with a new parameter. First we define the sixth-order method and then followed by the fifth-order method.

2.1. New Sixth-Order Method. The new sixth-order method for finding multiple root of a nonlinear equation is expressed as

$$
\begin{gathered}
y_{n}=x_{n}-m \frac{f\left(x_{n}\right)}{f^{\prime}\left(x_{n}\right)}, \\
z_{n}=x_{n}-m\left(\sum_{i=1}^{3} i\left(\frac{f\left(y_{n}\right)}{f\left(x_{n}\right)}\right)^{i / m}\right)\left(\frac{f\left(x_{n}\right)}{f^{\prime}\left(x_{n}\right)}\right), \\
x_{n+1}=z_{n}-m\left(\sum_{i=1}^{3} i\left(\frac{f\left(y_{n}\right)}{f\left(x_{n}\right)}\right)^{i / m}\right)^{2}\left(\frac{f\left(z_{n}\right)}{f\left(x_{n}\right)}\right)^{m^{-1}} \\
\times\left(\frac{f\left(x_{n}\right)}{f^{\prime}\left(x_{n}\right)}\right),
\end{gathered}
$$

where $n \in \mathbb{N}, x_{0}$ is the initial value provided that the denominators of (6) and (7) are not equal to zero.

Theorem 4. Let $\alpha \in I$ be a multiple root of multiplicity $m$ of a sufficiently differentiable function $f: I \subset \mathbb{R} \rightarrow \mathbb{R}$ for an open interval I. If $x_{0}$ is sufficiently close to $\alpha$, then the order of convergence of the new method defined by (10) is six.

Proof. Let $\alpha$ be a multiple root of multicity $m$ of a sufficiently smooth function $f(x), e=x-\alpha$ and $\hat{e}=y-\alpha$, where $y$ is defined in (6). have

Using the Taylor expansion of $f(x)$ and $f^{\prime}(x)$ about $\alpha$, we

$$
\begin{aligned}
f\left(x_{n}\right)= & \left(\frac{f^{(m)}(\alpha)}{m !}\right) e_{n}^{m}\left[1+c_{1} e_{n}+c_{2} e_{n}^{2}+c_{3} e_{n}^{3}+\cdots\right], \\
f^{\prime}\left(x_{n}\right)= & \left(\frac{f^{(m)}(\alpha)}{(m-1) !}\right) e_{n}^{m-1} \\
& \times\left[1+\left(\frac{m+1}{m}\right) c_{1} e_{n}+\left(\frac{m+2}{m}\right) c_{2} e_{n}^{2}+\cdots\right],
\end{aligned}
$$

where $n \in \mathbb{N}$ and

$$
c_{k}=\frac{m ! f^{(m+k)}(\alpha)}{(m+k) ! f^{(m)}(\alpha)} .
$$

From (6), we have

$$
\begin{aligned}
y_{n} & =e_{n}-m \frac{f\left(x_{n}\right)}{f^{\prime}\left(x_{n}\right)} \\
& =e_{n}-e_{n}\left[1-\frac{c_{1}}{m} e_{n}+\frac{(m+1) c_{1}^{2}-2 m c_{2}}{m^{2}} e_{n}^{2}+\cdots\right] .
\end{aligned}
$$

The expansion of $f\left(y_{n}\right)$ and about $\alpha$ is given by

$$
f\left(y_{n}\right)=\left(\frac{f^{(m)}(\alpha)}{m !}\right) \widehat{e}_{n}^{m}\left[1+c_{1} \widehat{e}_{n}+c_{2} \widehat{e}_{n}^{2}+c_{3} \widehat{e}_{n}^{3}+\cdots\right] .
$$

By using (11) and (15), we have

$$
\begin{aligned}
\left(\frac{f\left(y_{n}\right)}{f\left(x_{n}\right)}\right)^{m^{-1}}= & e_{n}\left(\frac{c_{1}}{m}\right) \\
\times & {\left[1+\left(2 m c_{2}-(m+2) c_{1}^{2}\right) m^{-1} e_{n}^{2}\right.} \\
& +2^{-1}\left(\left(2 m^{2}+7 m+7\right) c_{1}^{3}-2 m(3 m+7)\right. \\
& \left.\left.\times c_{1} c_{2}+6 m^{2} c_{3}\right) m^{-3} e_{n}^{3}+\cdots\right] .
\end{aligned}
$$

Since from (7) we have

$$
\begin{gathered}
z_{n}=e_{n}-m\left(\sum_{i=1}^{3} i\left(\frac{f\left(y_{n}\right)}{f\left(x_{n}\right)}\right)^{i / m}\right)\left(\frac{f\left(x_{n}\right)}{f^{\prime}\left(x_{n}\right)}\right), \\
f\left(z_{n}\right)=\left(\frac{f^{(m)}(\alpha)}{m !}\right) z_{n}^{m}\left[1+c_{1} z_{n}+\cdots\right] .
\end{gathered}
$$

As before we expand $f\left(z_{n}\right),\left(f\left(z_{n}\right) f\left(x_{n}\right)^{-1}\right)^{m^{-1}}$ and substitute the appropriate expressions in (10). After simplification we obtain the error equation

$$
\begin{aligned}
e_{n+1}= & 2^{-2} m^{-5} c_{1}\left(m c_{1}^{2}+3 c_{1}^{2}-2 m c_{2}\right) \\
& \times\left(m c_{1}^{2}+c_{1}^{2}-2 m c_{2}\right) e_{n}^{6}+\cdots .
\end{aligned}
$$

The error equation (18) establishes the sixth-order convergence of the new method defined by (10). 
2.2. New Fifth-Order Method. The new fifth-order method for finding multiple root of a nonlinear equation is expressed as

$$
\begin{gathered}
y_{n}=x_{n}-m \frac{f\left(x_{n}\right)}{f^{\prime}\left(x_{n}\right)}, \\
z_{n}=x_{n}-m\left(\sum_{i=1}^{3} i\left(\frac{f\left(y_{n}\right)}{f\left(x_{n}\right)}\right)^{i / m}\right)\left(\frac{f\left(x_{n}\right)}{f^{\prime}\left(x_{n}\right)}\right), \\
x_{n+1}=z_{n}-m\left(\frac{f\left(z_{n}\right)}{f\left(x_{n}\right)}\right)^{m^{-1}}\left(\frac{f\left(x_{n}\right)}{f^{\prime}\left(x_{n}\right)}\right),
\end{gathered}
$$

where $n \in \mathbb{N}, x_{0}$ is the initial value provided that the denominators of (19)-(21) are not equal to zero.

Theorem 5. Let $\alpha \in I$ be a multiple root of multiplicity $m$ of a sufficiently differentiable function $f: I \subset \mathbb{R} \rightarrow \mathbb{R}$ for an open interval I. If $x_{0}$ is sufficiently close to $\alpha$, then the order of convergence of the new method defined by (21) is five.

Proof. Substituting appropriate expressions in (21) and after simplification, we obtain the error equation

$$
e_{n+1}=m^{-4} c_{1}^{2}\left(m c_{1}^{2}+3 c_{1}^{2}-2 m c_{2}\right) e_{n}^{5}+\cdots .
$$

The error equation (22) establishes the fifth-order convergence of the new method defined by (21).

\section{Conclusion}

In this paper, we have introduced two new higher-order methods for solving nonlinear equations with multiple roots. Convergence analysis proves that the new methods preserve their order of convergence. The fifth-order method presented in this paper was actually the first improvement of the fourth-order method recently introduced in [8], and further improvement was made to achieve the sixth-order method. Simply by introducing new parameters we have achieved higher order of convergence. The purpose of this paper is to introduce higher order of convergence methods. Since we have verified the order of convergence of the new methods, we have not illustrated the numerical performance of these methods. Finally, we conjecture that these new higher-order iterative methods may be extended to an optimal eighthorder of convergence and may be considered a very good alternative to the classical methods given in [1-8].

\section{Acknowledgment}

The author is grateful to the reviewer for his helpful comments on this paper.

\section{References}

[1] S. Kumar, V. Kanwar, and S. Singh, "On some modified families of multipoint iterative methods for multiple roots of nonlinear equations," Applied Mathematics and Computation, vol. 218, no. 14, pp. 7382-7394, 2012.
[2] S. G. Li, L. Z. Cheng, and B. Neta, "Some fourth-order nonlinear solvers with closed formulae for multiple roots," Computers and Mathematics with Applications, vol. 59, no. 1, pp. 126-135, 2010.

[3] J. Peng and Z. Zeng, "An approach finding multiple roots of nonlinear equations or polynomials," Advanced Research on Electronic Commerce, Web Application, and Communication, vol. 149, pp. 83-87, 2012.

[4] E. Schröder, "Ueber unendlich viele Algorithmen zur Auflösung der Gleichungen," Mathematische Annalen, vol. 2, no. 2, pp. 317-365, 1870.

[5] J. R. Sharma and R. Sharma, "Modified Jarratt method for computing multiple roots," Applied Mathematics and Computation, vol. 217, no. 2, pp. 878-881, 2010.

[6] L. Shengguo, L. Xiangke, and C. Lizhi, "A new fourth-order iterative method for finding multiple roots of nonlinear equations," Applied Mathematics and Computation, vol. 215, no. 3, pp. 1288-1292, 2009.

[7] R. Thukral, "A new third-order iterative method for solving nonlinear equations with multiple roots," International Journal of Mathematics and Computation, vol. 6, pp. 61-68, 2010.

[8] Rajinder Thukral, "New fourth-order nonlinear solver for multiple roots," Advances in Numerical Analysis. Submitted in Advances in Numerical Analysis.

[9] W. Gautschi, Numerical Analysis: An Introduction, Birkhäuser, Boston, Mass, USA, 1997.

[10] J. F. Traub, Iterative Methods for Solution of Equations, Chelsea Publishing, New York, NY, USA, 1977.

[11] H. T. Kung and J. F. Traub, "Optimal order of one-point and multipoint iteration," Journal of the Association for Computing Machinery, vol. 21, pp. 643-651, 1974. 


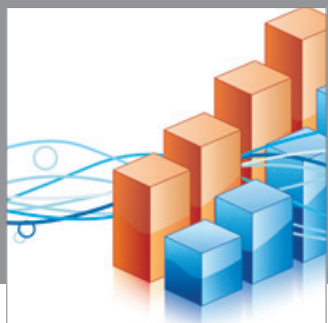

Advances in

Operations Research

mansans

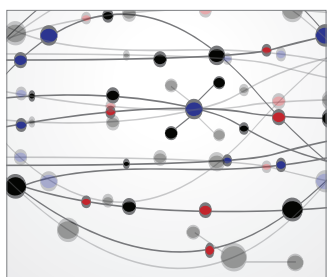

The Scientific World Journal
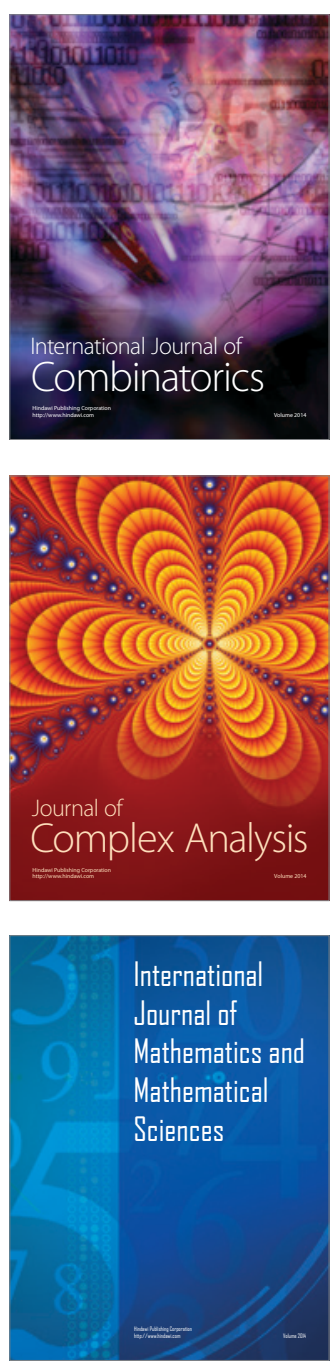
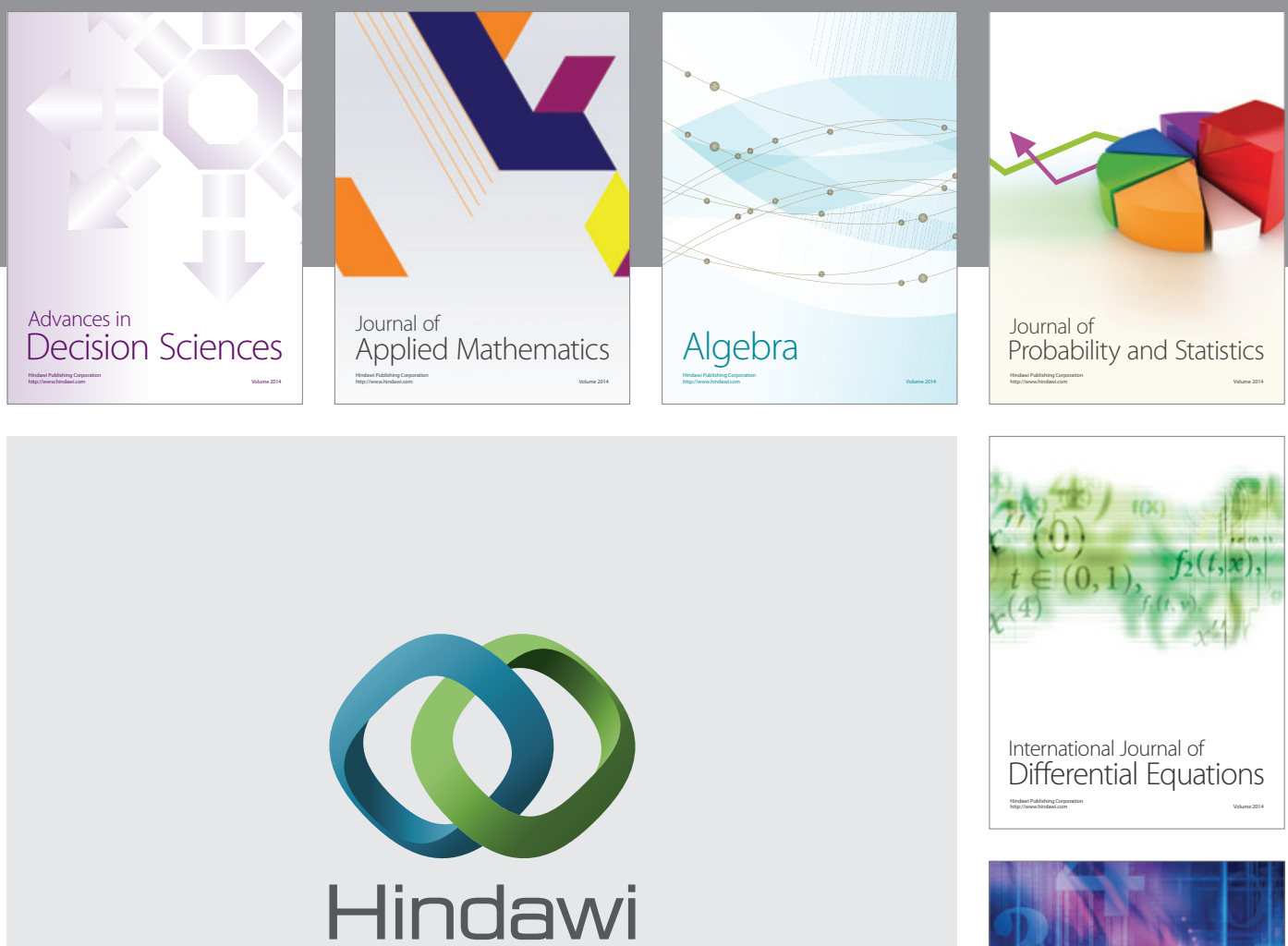

Submit your manuscripts at http://www.hindawi.com
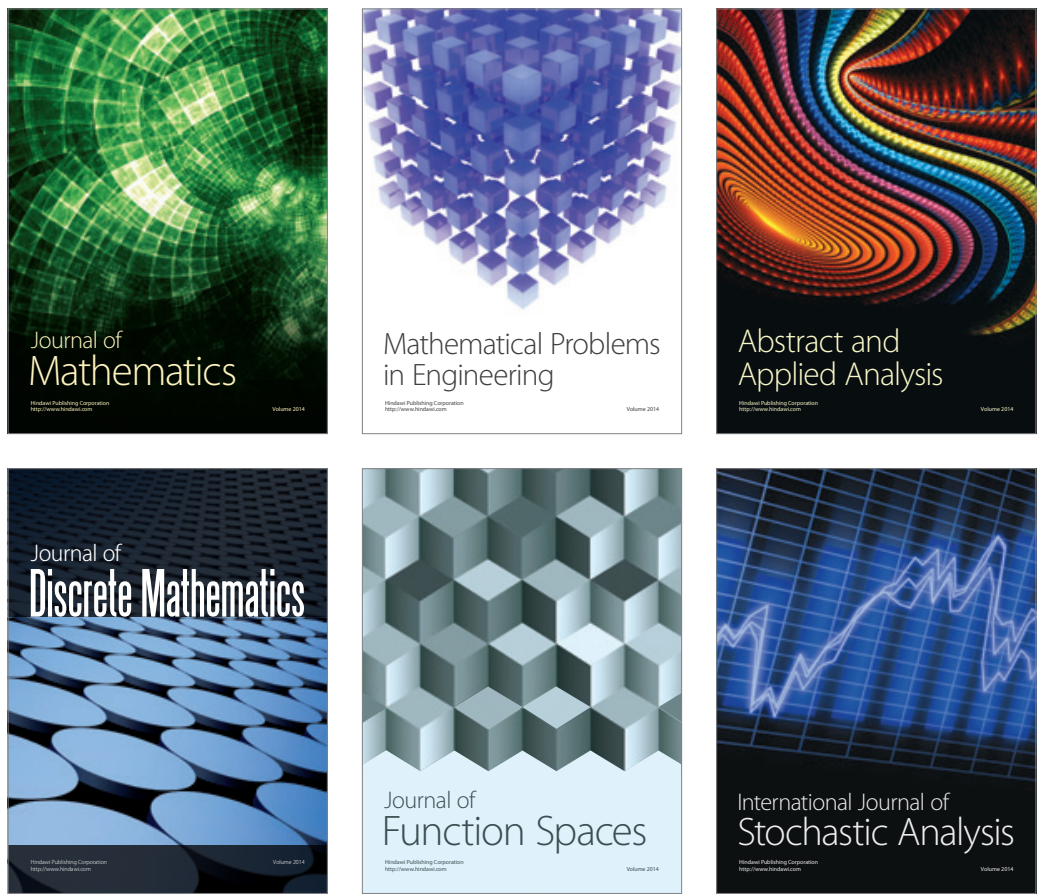

Journal of

Function Spaces

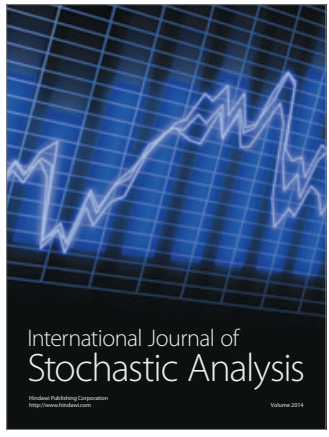

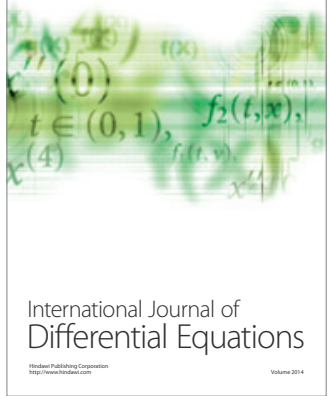
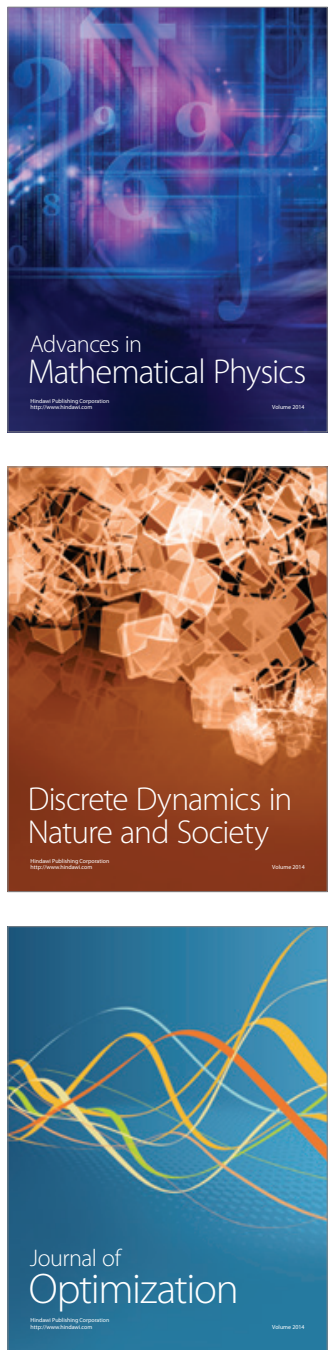